\title{
Vitamin intakes in Irish pre-school children aged 1-4 years
}

\author{
Á. Hennessy ${ }^{1}$, J. Walton ${ }^{1}$, B. McNulty ${ }^{2}$, A. Nugent ${ }^{2}$, M. Gibney ${ }^{2}$ and A. Flynn ${ }^{1}$, \\ Irish Universities Nutrition Alliance (iuna) at: \\ ${ }^{1}$ School of Food and Nutritional Sciences, University College Cork, Republic of Ireland, ${ }^{2}$ UCD Institute of Food and Health, \\ University College Dublin, Belfield, Dublin 4, Republic of Ireland
}

Early childhood is a critical time when dietary intake patterns are forming, which may impact on health in later life. The objective of this analysis was to estimate the mean daily vitamin intake of Irish pre-school children and was based on the National Pre-school Nutrition Survey (2010-2011), which was carried out to establish a database of habitual food and drink consumption in a representative sample of Irish pre-school children aged 1-4 years. A 4 day weighed food record was used to collect food intake data of 500 pre-school children. Analysis of dietary intake data was carried out using WISP ${ }^{\odot}$ (Tinuviel Software, Anglesey, UK), which contains McCance and Widdowson's The Composition of Foods ${ }^{(1)}$ and the Irish Foods Composition Database ${ }^{(2)}$.

The mean intake of selected vitamins and the percentage with intakes below the estimated average requirement (EAR) ${ }^{(3)}$, excluding under-reporters, are shown in Table 1 . BMR was calculated using standard equations ${ }^{(4)}$ and cut-off points, calculated as multiples of $\mathrm{BMR}^{(5)}$, were used to identify under-reporters (URs).

Table 1.

\begin{tabular}{|c|c|c|c|c|c|c|c|c|c|c|c|c|}
\hline & \multicolumn{3}{|c|}{1 year olds $(n=109)$} & \multicolumn{3}{|c|}{2 year olds $(n=93)$} & \multicolumn{3}{|c|}{3 year olds $(n=81)$} & \multicolumn{3}{|c|}{4 year olds $(n=96)$} \\
\hline & Mean & SD & $\%<$ EAR & Mean & SD & $\%<\mathrm{EAR}$ & Mean & SD & $\%<\mathrm{EAR}$ & Mean & SD & $\%<\mathrm{EAR}$ \\
\hline Vitamin A $(\mu \mathrm{g})$ & 787 & 497 & 4.6 & 639 & 354 & 20.4 & 712 & 398 & 14.8 & 632 & 345 & 13.5 \\
\hline Vitamin D $(\mu \mathrm{g})$ & 4.4 & 5.5 & - & 3.3 & 3.3 & - & 3.2 & 3.3 & - & 3.0 & 2.6 & - \\
\hline Thiamin (mg) & 1.0 & 0.4 & 0.0 & 1.2 & 0.4 & 0.0 & 1.1 & 0.3 & 0.0 & 1.2 & 0.3 & 0.0 \\
\hline Riboflavin (mg) & 1.7 & 0.7 & 0.0 & 1.7 & 0.6 & 0.0 & 1.6 & 0.5 & 0.0 & 1.6 & 0.5 & 0.0 \\
\hline Total Niacin (mg) & 18.1 & 5.6 & 0.0 & 21.9 & 6.0 & 0.0 & 22.2 & 5.8 & 0.0 & 23.0 & 6.3 & 0.0 \\
\hline Vitamin B6 (mg) & 1.3 & 0.6 & 0.0 & 1.5 & 0.6 & 0.0 & 1.6 & 0.5 & 0.0 & 1.6 & 0.6 & 0.0 \\
\hline Vitamin B12 $(\mu \mathrm{g})$ & 4.3 & 2.0 & 0.0 & 4.5 & 2.3 & 0.0 & 4.1 & 1.8 & 0.0 & 4.0 & 1.6 & 0.0 \\
\hline Total folate $(\mu \mathrm{g})$ & 165 & 81 & 0.0 & 186 & 68 & 0.0 & 199 & 88 & 0.0 & 198 & 81 & 0.0 \\
\hline Vitamin C (mg) & 80 & 45 & 0.0 & 87 & 68 & 2.2 & 87 & 48 & 1.2 & 96 & 53 & 1.0 \\
\hline
\end{tabular}

The main food contributors to vitamin intakes and their \% contribution are shown below.

Table 2.

\begin{tabular}{ll}
\hline Vitamin A & Milk \& Yogurt, 27\%; Vegetables \& Vegetable dishes, 25\%; Meat and Meat Products, 12\% \\
Vitamin D & Milk \& Yogurt, 30\%; Meat \& Meat Products, 17\%, Nutritional Supplements, $10 \%$ \\
Thiamin & Breakfast Cereals, 24\%; Milk \& Yogurt, 17\%; Meat and Meat Products, 13\% \\
Riboflavin & Milk \& Yogurt, 47\%; Breakfast Cereals, 20\%; Meat \& Meat Products, 7\% \\
Total Niacin & Meat \& Meat Products, 25; Breakfast Cereals, 18; Milk \& Yogurt 16\% \\
Vitamin B6 & Breakfast Cereals, 17\%; Meat \& Meat Products, 16\%; Milk \& Yogurt, 16\% \\
Vitamin B12 & Milk \& Yogurt, 58\%; Meat \& Meat Products, 13\%; Breakfast Cereals, 7\% \\
Folate & Milk \& Yogurt, 24\%; Breakfast Cereals, 21\%; Fruit \& Fruit Juices, $12 \%$ \\
Vitamin C & Fruit \& Fruit Juices, 43\%; Milk \& Yogurt, 16\%; Beverages, $10 \%$ \\
\hline
\end{tabular}

Intakes of most vitamins in the Irish pre-school population are adequate, with the exception of vitamins A and D. Over 78\% of 1-4 year olds had vitamin D intakes less than $5 \mu \mathrm{g} / \mathrm{d}$ and $20 \%$ had intakes less than $1 \mu \mathrm{g} / \mathrm{d}$. "Milk \& Yogurt", "Breakfast cereals", "Vegetables \& vegetable dishes", "Fruit \& fruit juices", "Meat and Meat Products" and "Beverages" were found to be the largest food contributors to selected vitamins in Irish pre-school children.

The project was funded by the Irish Government under the Food for Health Research Initiative 2007-2012.

1. Food Standards Agency. McCance \& Widdowson's The Composition of Foods Fifth \& Sixth Editions including supplemental volumes. Cambridge: Royal Society of Chemistry; 2002.

2. Black, L.J. Ireland J, Møller A, Roe M, Walton J, Flynn A, et al., Development of an on-line Irish food composition database for nutrients. J. Food Compos. Anal. 2011; 24(7): 1017-1023.

3. Department of Health UK. Dietary Reference Values of Food Energy and Nutrients for the United Kingdom. Report on Health and Social Subjects no. 41. London: H.M. Stationery Office; 1991.

4. Schofield, W.N., Predicting basal metabolic rate, new standards and review of previous work. Hum. Nutr. Clin. Nutr. 1985 ; 39 Suppl 1: 5-41.

5. Torun, B. Davies, PSW. Livingstone, MBE. Paolisso, M. Sackett, R \& Spurr, GB. Energy requirments and dietary energy recommendations for children and adolescents 1 to 18 years old. Eur J Clin Nutr 1996; 50, S37-S81. 